Synthesis of graphene-like transparent conductive films on dielectric substrates using a modified filtered vacuum arc system

Helge Lux', Peter Siemroth, Anna Sgarlata, Paolo Prosposito, Markus Andreas Schubert, Mauro Casalboni, and Sigurd Schrader

Citation: Journal of Applied Physics 117, 195304 (2015); doi: 10.1063/1.4921448

View online: http://dx.doi.org/10.1063/1.4921448

View Table of Contents: http://aip.scitation.org/toc/jap/117/19

Published by the American Institute of Physics

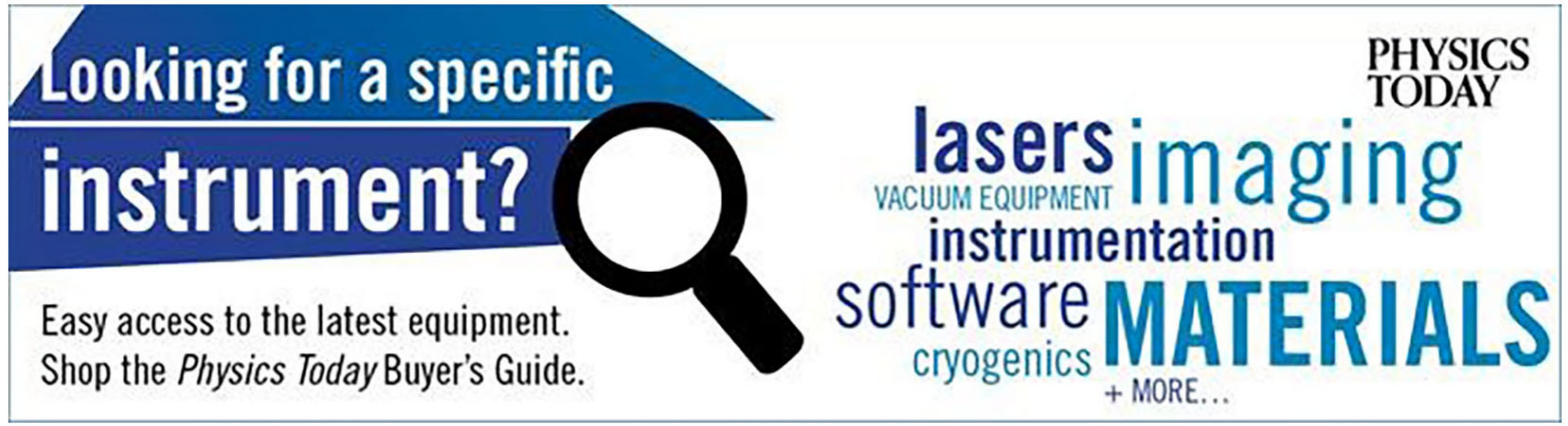




\title{
Synthesis of graphene-like transparent conductive films on dielectric substrates using a modified filtered vacuum arc system
}

\author{
Helge Lux, ${ }^{1, a)}$ Peter Siemroth, ${ }^{2}$ Anna Sgarlata, ${ }^{3}$ Paolo Prosposito, ${ }^{4}$ \\ Markus Andreas Schubert, ${ }^{5}$ Mauro Casalboni, ${ }^{4}$ and Sigurd Schrader ${ }^{1}$ \\ ${ }^{1}$ Technical University of Applied Sciences Wildau, Hochschulring 1, Wildau 15745, Germany \\ ${ }^{2}$ Arc Precision GmbH, Schwartzkopffstraße 2, Wildau 15745, Germany \\ ${ }^{3}$ Department of Physics, University of Roma - Tor Vergata, Via della Ricerca Scientifica 1, Roma 00133, Italy \\ ${ }^{4}$ Department of Industrial Engineering, University of Roma - Tor Vergata, and Italian Interuniversity \\ Consortium on Materials Science and Technology (INSTM), Research Unit Roma Tor Vergata Via del \\ Politecnico 1, Roma 00133, Italy \\ ${ }^{5}$ IHP Innovations for High Performance Microelectronics, Im Technologiepark 25, Frankfurt (Oder) 15236, \\ Germany
}

(Received 8 January 2015; accepted 9 May 2015; published online 20 May 2015)

\begin{abstract}
Here, we present a reliable process to deposit transparent conductive films on silicon oxide, quartz, and sapphire using a solid carbon source. This layer consists of partially ordered graphene flakes with a lateral dimension of about $5 \mathrm{~nm}$. The process does not require any catalytic metal and exploits a high current arc evaporation (Ф-HCA) to homogeneously deposit a layer of carbon on heated substrates. A gas atmosphere consisting of Argon or Argon/Hydrogen blend acting as a buffer influences the morphology of the growing film. scanning tunneling microscopy, transmission electron microscopy, and Raman spectra were used for a thorough characterization of the samples in order to optimize the growth parameters. The best carbon layers have a surface resistance of $5.7 \times 10^{3} \Omega_{\square}$ whereas the optical transparency of the coatings is $88 \%$ with an excellent homogeneity over areas of several $\mathrm{cm}^{2}$. Such results are compatible with most semiconductor fabrication processes and make this method very promising for various industrial applications. C 2015 AIP Publishing LLC. [http://dx.doi.org/10.1063/1.4921448]
\end{abstract}

\section{INTRODUCTION}

Graphene, the single monolayer modification of $\mathrm{sp}^{2}$ hybridized carbon, has attracted much interest as a new material for microelectronics and as a substitute for transparent conductive oxides (TCO). ${ }^{1,2}$ However, there is still no reliable fabrication process of graphene as a TCO-substituent with sufficient transmission and conductivity, neither for high-quality monolayer graphene nor for any graphenecontaining film.

Generally, the transfer process of graphene is the most common adopted procedure. The graphene films grown on the metal surface are lifted-off by a suitable carrier foil and subsequently transferred onto the device. This complex and expensive process can damage the coating and can potentially leave contaminations on the graphene (substrate material, polymers from the carrier of the transfer process, or organic solvents). The great challenge should be the deposition of the coating directly on the insulating substrates. One of the most promising methods to produce transparent carbon coatings is the chemical method based on reduction of graphene oxide (RGO). ${ }^{3}$ Such deposition processes have broad applications in the field of bio-, gas-, and DNA-sensors. ${ }^{3}$ However, they require complex chemical processes and produce layers with poor electrical conductivity.

In this paper, we present an alternative approach which allows the growth of homogeneous large-area graphene-like

\footnotetext{
a) Author to whom correspondence should be addressed. Electronic mail: lux@th-wildau.de, Tel.: +00 493375508119
}

coatings directly on a dielectric substrate without any catalytic metal film with good electrical and optical behavior. The coatings cannot be classified as "graphene layers," since their thickness is several nanometers. However, we define them as "graphene-like" because all the observables (Raman signal, transparency, and conductivity) originate from 2-D graphene flakes.

The basic idea of the process described here consists of the thermal transformation of a thin carbon layer into its $\mathrm{sp}^{2}$ hybridized form during the deposition of carbon atoms/ions from a modified high current arc source, originally developed for the production of ultra-hard carbon films (tetrahedral amorphous carbon, ta-C). The main reason of its exceptional hardness is the high percentage of sp3-bonds, caused by the so-called subplantation of the energetic carbon ions into the growing film. ${ }^{4,5}$ In the present arrangement, the impinging energy of the carbon ions is reduced in order to avoid deep implantation into the substrate material, and instead to promote a gentle deposition of them in the first atomic layer. Simultaneous heating of the substrate provide slight mobility to the deposited atoms, and allows them to self-organize into a predominantly $\mathrm{sp}^{2}$-bonded structure. In literature, only a few publications can be found dealing with the production of conductive carbon coatings using arc evaporation. Ilie et al. ${ }^{6}$ and Ferrari et al. ${ }^{7}$ reported on a method of ex-post heating of a highly $\mathrm{sp}^{3}$-hybridized amorphous carbon film, resulting in a strongly reduced resistivity. However, the Raman measurements of the treated coatings provided a quite constant $\mathrm{sp}^{3}$-content of the films when the temperature 


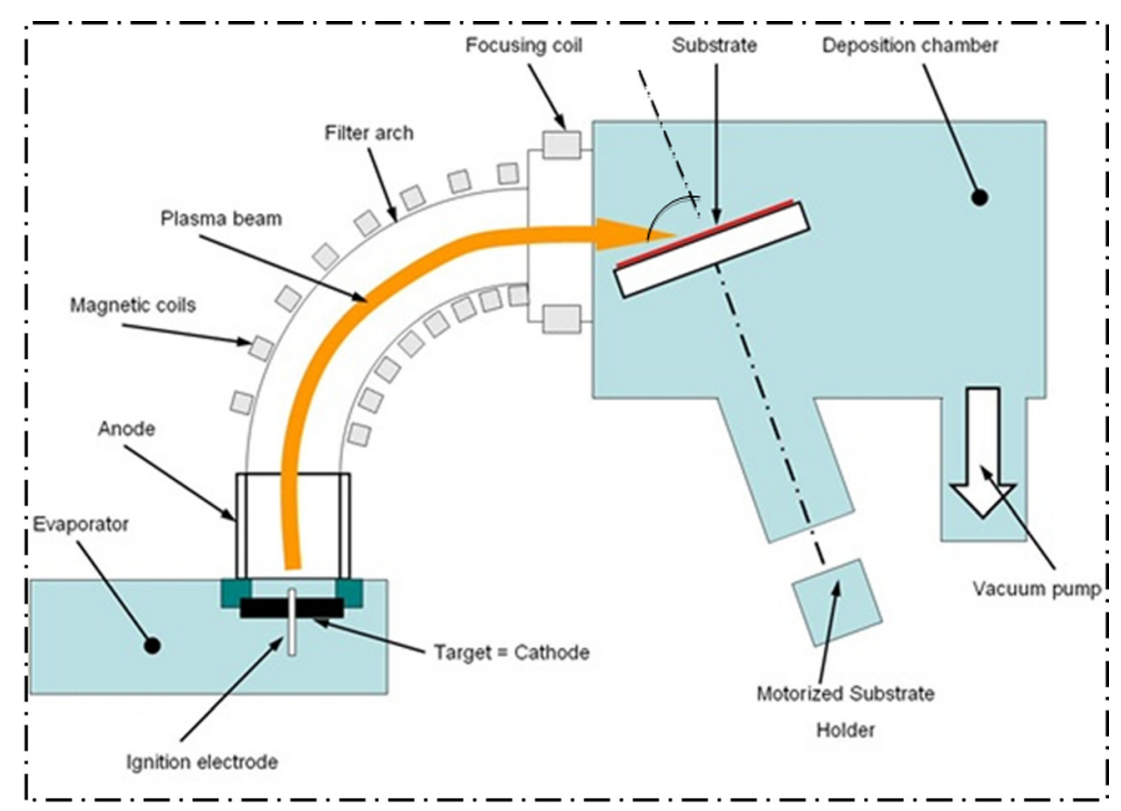

FIG. 1. Filter arc setup for carbon deposition. was lower than $1500{ }^{\circ} \mathrm{C}$ (cf. also Ref. 8). Therefore, it seems impossible to convert amorphous carbon into a predominantly $\mathrm{sp}^{2}$-bonded carbon only by ex-post heating unless using higher temperatures incompatible with glass or quartz substrates. Many publications have already been published dealing with the graphene production, using low-current arc evaporation under relatively high pressure. ${ }^{9}$ These processes normally produce multilayered graphene-flakes, which can be used, e.g., in dispersions for conductive spayed coatings. The resulting carbon-black-coatings consist of unordered graphene flakes with no particularly optical transmission (due to high thickness), and therefore, they have to be carefully distinguished from our graphene-like films. An alternative process based on the heating of the substrate during the arc process ${ }^{10}$ produced a predominantly perpendicular graphitic growth, as theoretically confirmed by Marks et al. ${ }^{11}$ that should be avoided for its deleterious effect on sheet resistance.

The process described here can be controlled by means of several external parameters such as pressure, composition of buffer gas, substrate temperature, impinging angle, and deposition rate. All of these parameters can influence the growth conditions and allow the optimization to produce transparent and conductive carbon coatings of good quality.

\section{EXPERIMENTAL}

The current filtered high current arc ( $\Phi-H C A)$ set-up is shown in Figure 1 and described in detail in Refs. 12 and 13. The carbon cathode (inside the evaporator) is surrounded by a cylindrical, water-cooled anode. The evaporator works in a pulsed mode using $1.0 \mathrm{~ms}$ pulses with an arc current up to 3000 A. To avoid thermal spikes as well as excessively deep penetration of carbon ions into the substrate, the impact particle energy has been optimized. For this purpose, two methods have been used simultaneously. First, the ions are guided through a noble gas atmosphere in order to reduce their kinetic energy by collisions with the gas atoms, and second, the sample is tilted in order to reduce the effective impact energy. Increasing the angle between the normal to the sample surface and incident beam, the penetration depth decreases. Best conditions were obtained for an angle of incidence of $70^{\circ}$ (see Figure 1). The noble gas pressure was changed in a range between $0.1 \mathrm{~Pa}$ and $150 \mathrm{~Pa}$.

However, it has to be noted that the dominant factor, which decreases the kinetic energy of carbon ions, is the noble gas atmosphere, whereas the influence of the substrate tilt is limited. All parameters used for the current work are a result of a systematic optimization process and are summarized in Table I.

Quartz, silicon oxide, and sapphire plates were used as insulating substrate materials. In order to measure the optical transmittance in a wide range of wavelength (from UV to infrared), the quartz substrate was used.

For each sample, the sheet resistance and the Hall mobility of the charge carriers were measured using a Hall measurement device with a 4-tip arrangement and a magnetic field of $1 \mathrm{~T}$ (Lakeshore LS 4005). The transmission was determined by means of a spectrophotometer (Perkin Elmer Lambda 2000). The Raman spectra were measured using a WITec alpha 300 confocal Raman microscope with an excitation wavelength of $\lambda=488 \mathrm{~nm}$. To study the surface quality as well as the carbon structure and layer thickness, scanning tunneling microscopy (STM) and transmission electron microscopy (TEM) were used. The STM

TABLE I. Optimized parameters for the deposition of transparent and conductive carbon coatings.

\begin{tabular}{lc}
\hline \hline Parameter & Value \\
\hline Cathodic arc current & $2500 \mathrm{~A}$ \\
Arc duration time & $1 \mathrm{~ms}$ \\
Magnetic filter current & $200 \mathrm{~A}$ \\
Plasma pulse frequency & $0.66 \mathrm{~Hz}$ \\
Noble gas pressure (argon) & $100 \mathrm{~Pa}$ \\
Hydrogen gas pressure (optional) & $20 \mathrm{~Pa}$ \\
Substrate temperature & $950^{\circ} \mathrm{C}$ \\
Substrate tilt angle & $70^{\circ}$ \\
\hline \hline
\end{tabular}




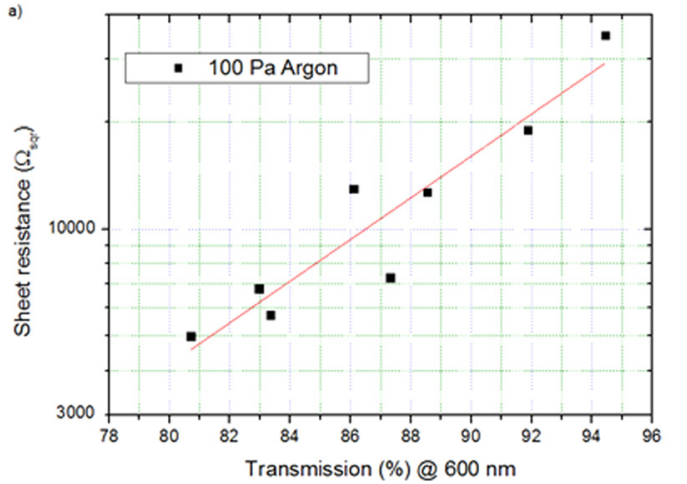

measurements were carried out using a WA-Technology microscope with a tungsten tip. The topographic images were acquired in constant-current mode, and the tunneling conditions were optimized in order to attain the highest resolution. TEM samples have been conventionally prepared by grinding, polishing, and argon ion milling. For TEM measurements, we used a Tecnai Osiris from FEI operated at $200 \mathrm{kV}$.

\section{RESULTS AND DISCUSSION}

\section{A. Coating under noble gas atmosphere}

In order to characterize our samples, we performed measurements of sheet resistance $\left(\left[\mathrm{R}_{\mathrm{s}}\right]=\Omega_{\square}\right)$ and optical transmission at a wavelength of $600 \mathrm{~nm}$ for several samples having different layer thicknesses. Figure 2(a) shows the correlation between transmission and sheet resistance. The data show a linear behaviour as expected for Graphene and Graphene-like coatings. ${ }^{2}$ This indicates that even for thin coatings (high transmission), no island growth occurs. A picture of a coated quartz sample showing the high homogeneity on the whole surface $\left(2.5 \times 2.5 \mathrm{~cm}^{2}\right)$ can be seen in Figure 2(b).

Figure 3(a) presents the Raman spectrum for a typical carbon coating on bare quartz. The well resolved and narrow $D$ and $G$ peaks at 1350 and $1530 \mathrm{~cm}^{-1}$, respectively, are present together with the $2 \mathrm{D}$ band at $2690 \mathrm{~cm}^{-1}$. These peaks are the typical signatures of a graphene-like coating. The strong $\mathrm{D}$ band shows the presence of disordered carbon atoms as well as a high density of grain boundaries.

For that reason, the $\mathrm{G}+\mathrm{D}$ band at $\sim 2950 \mathrm{~cm}^{-1}$ is also visible. The intensity ratio $\mathrm{I}_{\mathrm{D}} / \mathrm{I}_{\mathrm{G}}$ of 1.5 indicates a distance between the defects (grain size) of about $5 \mathrm{~nm} .{ }^{14}$ The position
FIG. 2. (a) Sheet resistance as a function of transmission at $600 \mathrm{~nm}$ wavelength of light. (b) Photograph of a homogeneous coated quartz plate. of the 2D-peak at $2690 \mathrm{~cm}^{-1}$ has shifted to a higher wavenumber with respect to that expected for a graphene monolayer on silicon oxide, ${ }^{15,16}$ which is a result of the layer thickness of several nanometers. The homogeneity of the deposition was investigated by measuring the intensity of the 2D-band over an area of $(100 \mu \mathrm{m} \times 100 \mu \mathrm{m})$ (Figure 3, inset). The resulting false color picture shows neither holes nor any partial inhomogeneity (the diagonal line in the upper left corner is an artifact of the measuring device that appears on every scan at the same location). The transmission spectrum of the same sample (Figure 3(b), black line) shows the typical graphene-like characteristics. In the visible range, the absorption is nearly independent on the wavelength. The local minimum at $270 \mathrm{~nm}$ is known as the van-Hove singularity, and it is directly related to the band structure. The transmission data fit well with a simulated spectrum of about 8-layer graphene on quartz (corresponding to layer thickness of approximately $3 \mathrm{~nm}$ ), modeled by using optical constants $(\mathrm{n}, \mathrm{k})$ published by Boosalis et al. ${ }^{17}$ In the UV range of the spectrum, the sample shows a stronger absorption than expected. This is probably caused by higher disorder compared with the pristine graphene. The disordered graphitic structures produce an intense Rayleigh scattering, which is responsible for the lower transmission below $350 \mathrm{~nm}$. The evident blue shift of the peak seems to confirm this hypothesis.

To investigate the surface quality as well as the carbon structure and layer thickness, STM and TEM measurements were performed. Figure 4(a) shows the STM micrograph of a typical high transmittance sample. The STM measurements reveal a rough and disordered carbon coating characterized by a fractal cauliflower-shape structure with an average roughness of $3.0 \mathrm{~nm}$ comparable to that published in Ref. 18.
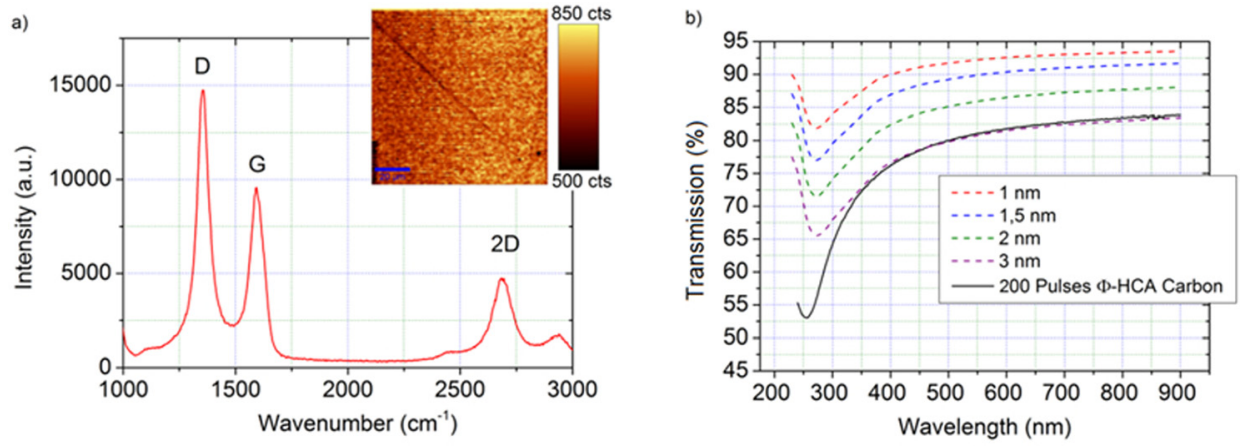

FIG. 3. (a) Raman spectrum of a graphene-like coating on bare quartz; inset: False color picture of $\mathrm{I}_{2 \mathrm{D}}$ (100 $\mu \mathrm{m} \times 100 \mu \mathrm{m})$. (b) Transmission spectra of a graphene-based coating on bare quartz ( 200 arc pulses, black line) in comparison to the modeled transmission of four different graphene thicknesses (dashed lines). 

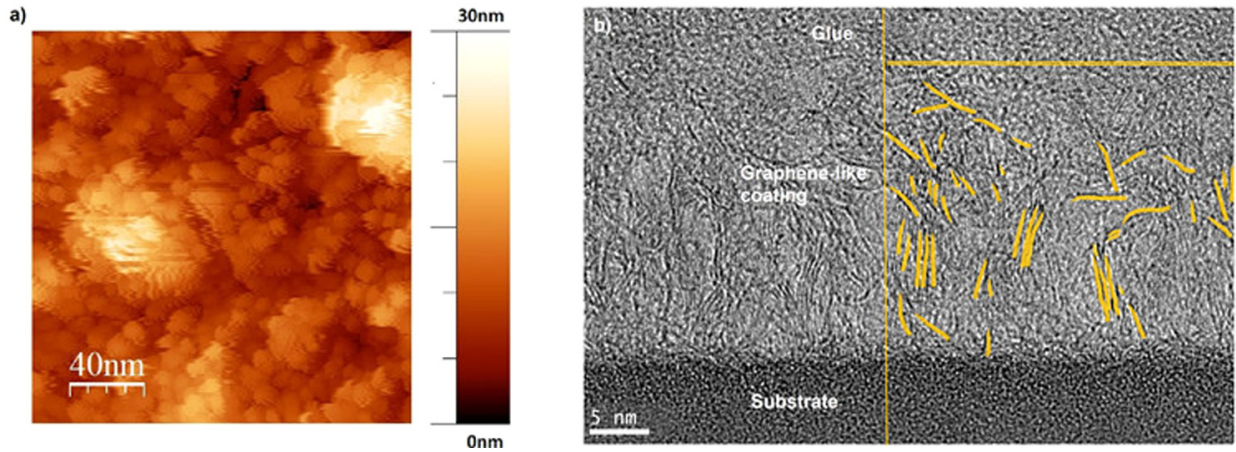

FIG. 4. (a) STM image of a sample with an optical transmission of $82 \%$. (b) TEM image of the cross section of the same sample.

TABLE II. Properties of a graphene-like carbon layer deposited by use of the modified $\Phi$-HCA technique at an argon pressure of $100 \mathrm{~Pa}$.

\begin{tabular}{lc}
\hline \hline R@T $=88.5 \%$ & $10.2 \mathrm{k} \Omega_{\square}$ \\
Electron mobility & $3.5 \mathrm{~cm}^{2} / \mathrm{Vs}$ \\
Thickness@T $=85 \%$ & $18 \mathrm{~nm}$ \\
Roughness, $\mathrm{R}_{\mathrm{a}}$ & $3.0 \mathrm{~nm}$ \\
Spec. resistivity & $1.8 \mu \Omega \mathrm{cm}$ \\
\hline \hline
\end{tabular}

On the same sample, a TEM characterization shown in Figure 4(b) has been carried out. On the right side of Figure 4(b), the graphene flakes are color-enhanced for better visibility. A thick carbon layer of about $25 \mathrm{~nm}$ is clearly visible. Along the first $10 \mathrm{~nm}$ upon the silicon oxide surface, the graphene flakes grow almost perpendicular with respect to the surface, as it has been already published. ${ }^{10}$ Above this first region, a mostly flat growth of the graphene sheets occurs. The grain size of about $5 \mathrm{~nm}$ observed in this picture is consistent to that calculated from the Raman spectrum and observed in the STM pictures. This result seems to be in contradiction with the measured optical absorption, which suggests a lower thickness of about $3 \mathrm{~nm}$ (see Figure 3(b)). However, these findings can be explained considering that the perpendicular growth of graphene fragments contributes to a lower extent to the absorption than the horizontal or quasi-horizontal layers when measured by optical transmission in normal incidence where the electric field of the light is oscillating along the substrate plane. We can conclude that

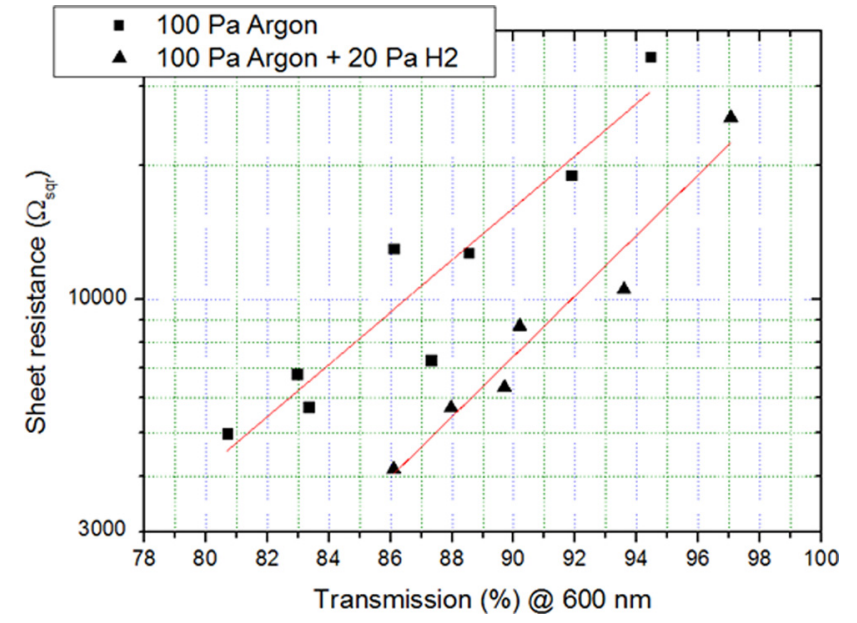

FIG. 5. Sheet resistance as a function of optical transmission at $600 \mathrm{~nm}$ of graphene-based coatings, formed using the current $\Phi$-HCA with hydrogen (triangles) and without hydrogen (squares). the domains grown vertically are responsible for the higher thickness and roughness, while the graphene-like Raman spectrum and the relatively high electrical conductivity are caused by the horizontal graphene growth of the upper layers. The electron mobility of this coating measured using a 4-tip Hall equipment is about $5 \mathrm{~cm}^{2} / \mathrm{Vs}$.

The properties of the investigated graphene-like carbon layers, deposited using the modified filtered arc technique at an argon pressure of $100 \mathrm{~Pa}$, are summarized in Table II.

\section{B. Influence of hydrogen on the deposition process}

Hydrogen is often used in CVD and PECVD processes on metals because of its potential for selective carbon etching. Therefore, it can improve the quality of the growing carbon film. In order to improve the deposition process and to avoid the perpendicular growth, the possibility of adding up to $20 \mathrm{~Pa}$ of hydrogen to the noble gas atmosphere $(100 \mathrm{~Pa}$ Argon) was tested. Samples of different thicknesses were prepared under these conditions. Figure 5 shows the plot of sheet resistance vs. transmission for such samples in comparison to those prepared by means of previously described process without the use of hydrogen. Adding hydrogen to the Argon gas reduces the deposition rate significantly by one order of magnitude. This effect is probably caused by the formation of $\mathrm{C}_{\mathrm{x}} \mathrm{H}_{\mathrm{y}}$-compounds, which are hardly involved in the deposition process. As shown in Figure 5, the addition of hydrogen significantly increases the transmission.

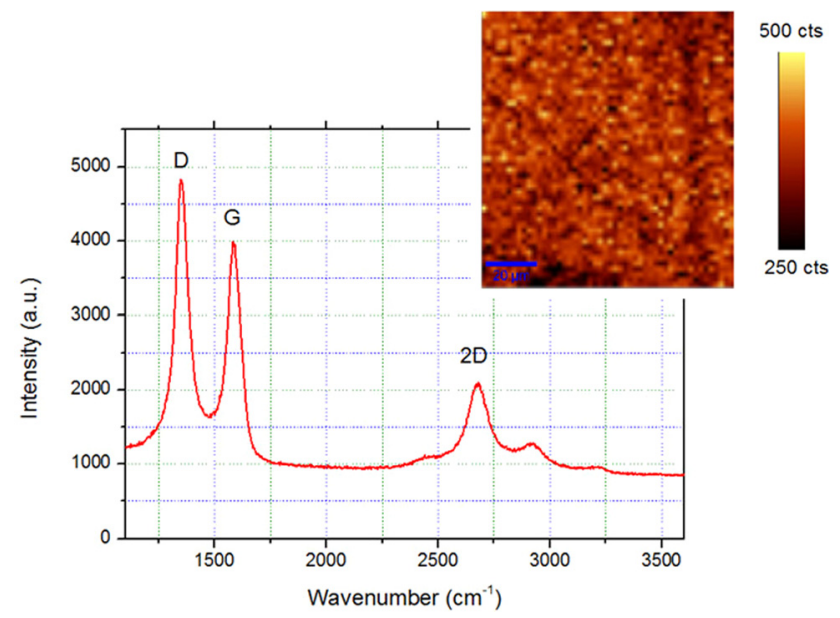

FIG. 6. Raman spectrum of a graphene-like coating on bare quartz, coated in hydrogen containing atmosphere. Inset shows a false color picture of $\mathrm{I}_{2 \mathrm{D}}$ $(100 \mu \mathrm{m} \times 100 \mu \mathrm{m})$. 


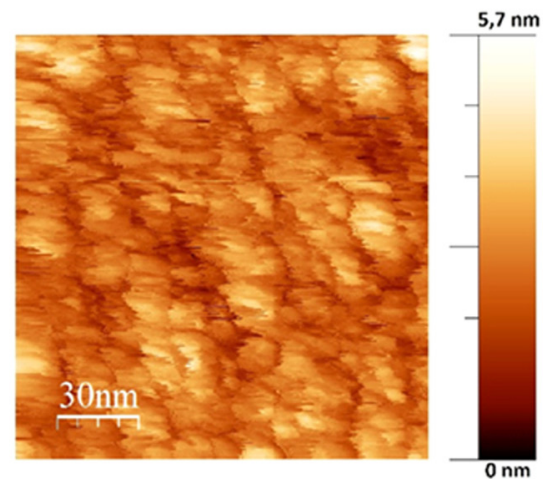

b)

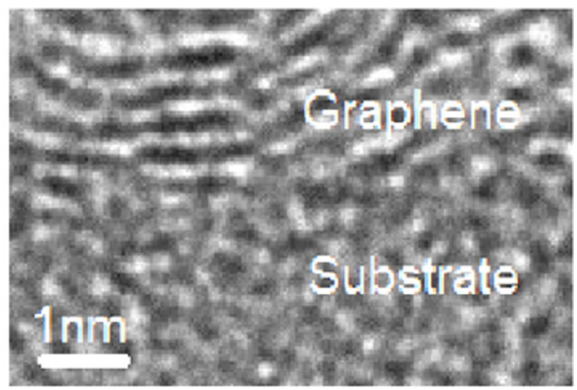

FIG. 7. (a) STM image of a graphenelike coating obtained in $\mathrm{H}$ Ar blend atmosphere with an optical transmission of $87 \%$. (b) TEM image of the cross section of the same sample.
Moreover, the electron mobility increases almost by one order of magnitude and was measured to be about $30 \mathrm{~cm}^{2} / \mathrm{Vs}$.

The referring Raman spectrum of the sample is shown in Figure 6. The Raman spectra of samples obtained with and without hydrogen are very similar except for slightly reduction of the $2 \mathrm{D}$-peak relative intensity. This underlines the limitations of Raman spectroscopy for characterizing a multi-crystalline, graphene-like film with different crystal orientations. The $\mathrm{I}_{\mathrm{D}} / \mathrm{I}_{\mathrm{G}}$ ratio of 1.2 provides the real graphene-like flake size of about $5 \mathrm{~nm}$, confirmed by TEM picture. Another important result is the excellent homogeneity of the coating that is not affected by the addition of hydrogen (see the inset in Figure 6).

The referring STM and TEM pictures of a sample with $87 \%$ optical transmission are shown in Figure 7 . One of the most relevant finding from the STM measurements is the decrease of the average surface roughness. The morphological structure of the carbon changes from cauliflower-shaped, unordered carbon (Fig. 4(a)) to a mostly flat and closed carbon film. Moreover, TEM picture of the same sample (Figure 7(b)) shows a completely different structure with respect to the previous one shown in Fig. 4(b).

The first four or five layers of graphene flakes are ordered, predominantly flat and parallel to the substrate. Consequently, the perpendicular growth can be avoided by the addition of hydrogen. As the deposition process proceeds and the coating is getting thicker, an unordered carbon growth occurs with a high percentage of perpendicular orientation.

The properties of the graphene-like carbon layer, deposited under a hydrogen containing atmosphere are summarized in Table III.

The main advantage of the above described process is the possibility to be employed on arbitrary substrates withstanding temperatures of about $800{ }^{\circ} \mathrm{C}$. Different insulating substrate materials were coated in hydrogen/argon atmosphere, using the optimized parameters described above. The process

TABLE III. Properties of a graphene-like carbon layer, deposited under hydrogen containing atmosphere (100 $\mathrm{Pa}$ argon $+20 \mathrm{~Pa}$ hydrogen $)$.

\begin{tabular}{lc}
\hline \hline $\mathrm{R} @ \mathrm{~T}=88 \%$ & $5.7 \mathrm{k} \Omega_{\square}$ \\
Electron mobility & $20.30 \mathrm{~cm}^{2} / \mathrm{Vs}$ \\
Thickness@T =87\% & $8.5 \mathrm{~nm}$ \\
Roughness, $\mathrm{R}_{\mathrm{a}}$ & $0.5 \mathrm{~nm}$ \\
Spec. resistivity & $0.4 \mu \Omega \mathrm{cm}$ \\
\hline
\end{tabular}

was tested on different insulating substrates, namely, sapphire and silicon, showing similar electrical and optical properties.

\section{Comparison with other deposition methods}

It is interesting to compare the electrical and optical properties of carbon based coatings produced with the current approach presented here with other established methods of graphene and reduced graphene oxide.

Compared to the measurements on transferred graphene deposited by a low-temperature PE-CVD process on copper (Figure 8, blue dashed line), the sheet resistances achieved in the present study are significantly lower if a hydrogen containing atmosphere is used (red line). The reported results on annealed RGO (purple dashed line) are in the same range of our coatings. The resistivity of exfoliated high-quality graphene is much lower (green dashed line), as a result of the much larger grain size (lower density of grain boundaries) and the lower distortions as well. One of the main advantages of the approach presented here is the good tuneability of both the optical transmission and the sheet resistance due to the pulsed deposition process that allows an optimal control of the thickness through the number of arc pulses provided maintaining fixed all the other process parameters.

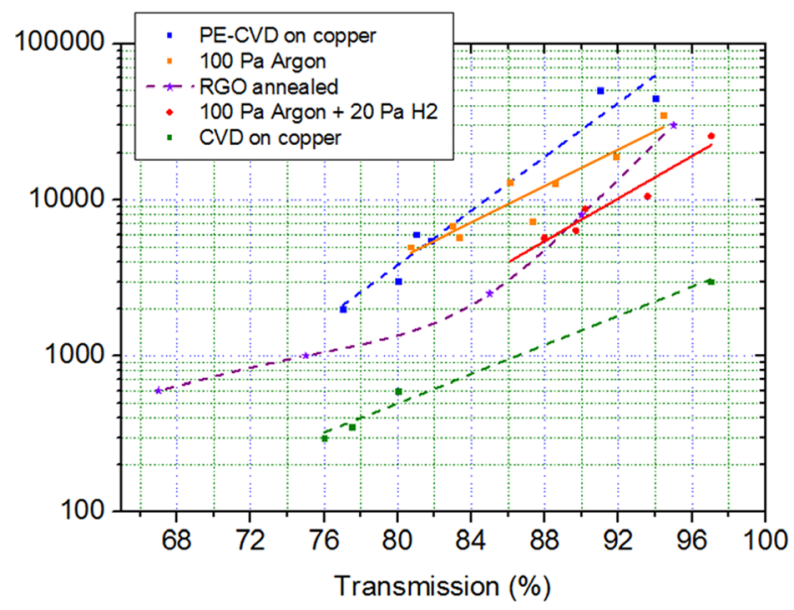

FIG. 8. Sheet resistance vs. transmission of carbon coatings measured at light wavelength of $600 \mathrm{~nm}$, which have been deposited using different processes. Coatings from this work (pure argon atmosphere-orange line), lowtemperature PE-CVD on copper (blue dashed line), ${ }^{20}$ coatings from this work (argon/hydrogen atmosphere-red line), annealed RGO (purple dashed line, ${ }^{21}$ and transferred high-quality graphene grown on copper (green dashed line). ${ }^{2}$ 


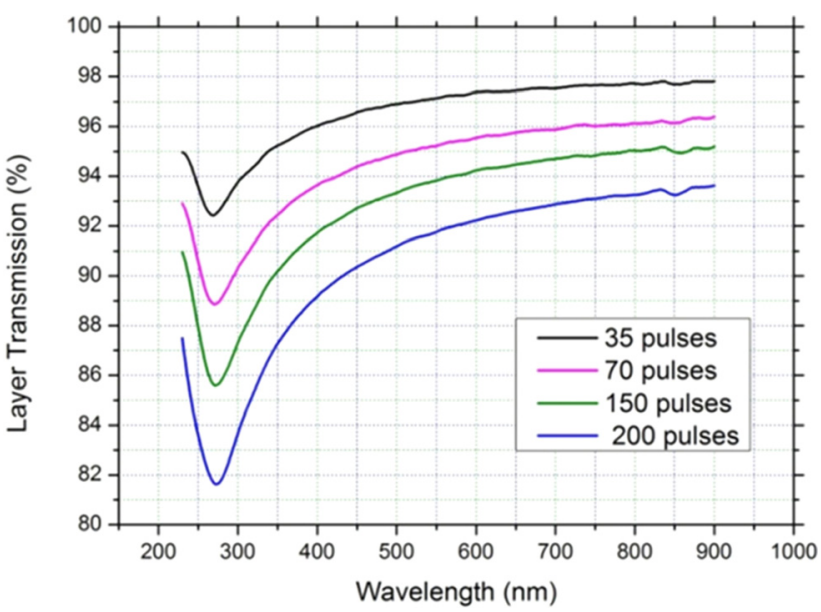

FIG. 9. Transmission spectra for different samples obtained with an increasing number of arc pulses.

Furthermore, this process does not require any catalytic metal and no transfer process as well. To illustrate the scalability of the process, Figure 9 shows the transmission spectra of different graphene-like coatings for different numbers of arc pulses.

Regarding the electron mobility, the measured values between $3 \mathrm{~cm}^{2} / \mathrm{Vs}$ and $30 \mathrm{~cm}^{2} / \mathrm{Vs}$ are much lower than the reported measurements on a freestanding graphene layer $\left(200000 \mathrm{~cm}^{2} / \mathrm{Vs}\right) .^{19}$ The electron mobility substantially depends on the grain size (density of grain boundaries) as well as on the crystal orientation (flat or perpendicular growth) and on substrate effects. However, it has to be noted that, in spite of the relatively low electron mobility, this material could be efficiently used as transparent conductive material in substitution of indium tin oxide (ITO) or zinc oxide for some specific application such as touch screen and EMI shield.

\section{CONCLUSION}

A new completely metal free deposition process for graphene-like thin layers is presented. A filtered vacuum arc system using argon and argon/hydrogen blend with low deposition rate was used to deposit coatings directly on different insulating substrates with excellent homogeneity on large areas. The resulting thickness can be adjusted easily by the number of arc pulses. Furthermore, for sample deposited under argon/hydrogen atmosphere, a perpendicular growth of the graphitic structure can be effectively avoided.

The obtained coatings have a sheet resistance between $5 \mathrm{k} \Omega_{\square}$ and $20 \mathrm{k} \Omega_{\square}$, depending on the number of layers. The transparency is in the range of $85 \%-95 \%$ at $600 \mathrm{~nm}$. These figures are strongly promising for the possible use of such process in the production of transparent electrodes. Moreover, the required materials, the way of deposition, and the involved temperature could be potentially compatible with other fabrication technologies.

\section{ACKNOWLEDGMENTS}

The authors gratefully acknowledge the valuable support of the Graphene Research Group of the Leibnitz-Institute for
Innovative Microelectronics (IHP) in Frankfurt (Oder), Germany. The authors also thank Dr. J. Bauer, Technical University of Applied Sciences Wildau, Germany for his help in modeling and calculating the optical constants of graphene. This work was funded by the Ministry of Science, Research and Culture (MWFK) of the Federal State of Brandenburg, Germany, in the frame of the Social Fund for Economic Development (ESF) of the European Union under contact number 1332537.

${ }^{1}$ K. S. Novoselov, V. I. Falko, L. Colombo, P. R. Gellert, M. G. Schwab, and K. Kim, “A Roadmap for graphene," Nature 490, 192-200 (2012).

${ }^{2}$ F. Bonaccorso, Z. Sun, T. Hasan, and A. C. Ferrari, "Graphene photonics and optoelectronics," Nat. Photonics 4, 611 (2010).

${ }^{3}$ D. Chen, H. Feng, and J. Li, "Graphene oxide: Preparation, functionalization, and electrochemical applications," Chem. Rev. 112(11), 6027-6053 (2012).

${ }^{4}$ Th. Frauenheim, G. Jungnickel, Th. Köhler, and U. Stephan, "Non-crystalline solids structure and electronic properties of amorphous carbon: From semimetallic to insulating behaviour," J. Non Cryst. Solids 182, 186-197 (1995).

${ }^{5}$ Y. Lifshitz, S. R. Kasi, and J. W. Rabalais, "Subplantation model for film growth from hyperthermal species: Application to diamond," Phys. Rev. Lett. 62, 1290-1293 (1989).

${ }^{6}$ A. Ilie, A. C. Ferrari, T. Yagi, S. E. Rodil, and J. Robertson, "Role of $\mathrm{sp}^{2}$ phase in field emission from nanostructured carbons," J. Appl. Phys. 90, 2024 (2001)

${ }^{7}$ A. C. Ferrari, B. Kleinsorge, N. A. Morrison, A. Hart, V. Stolojan, and J. Robertson, "Stress reduction and bond stability during thermal annealing of tetrahedral amorphous carbon," J. Appl. Phys. 85, 7191 (1999).

${ }^{8}$ D. S. Grierson, A. V. Sumant, A. Konicek, T. A. Friedmann, J. P. Sullivan, and R. W. Carpick, "Thermal stability and rehybridization of carbon bonding in tetrahedral amorphous carbon," J. Appl. Phys. 107, 033523 (2010).

${ }^{9}$ B. Shen, J. Ding, X. Yan, W. Feng, J. Li, and Q. Xue, "Influence of different buffer gases on synthesis of few-layered graphene by arc discharge method," Appl. Surf. Sci. 258, 4523-4531 (2012).

${ }^{10}$ Y. Yin, J. Zou, and D. R. Mckenzie, "Preferred orientation in carbon films induced by energetic condensation," Nucl. Instrum. Methods Phys. Res. B 119, 587 (1996).

${ }^{11}$ N. A. Marks, M. F. Cover, and C. Koce, "Simulating temperature effects in the growth of. tetrahedral amorphous carbon: The importance of infrequent events," Appl. Phys. Lett. 89, 131924 (2006).

${ }^{12}$ B. Petereit, P. Siemroth, H.-H. Schneider, and H. Hilgers, "High current filtered arc deposition for ultra-thin carbon overcoats on magnetic hard disks and read-write heads," Surf. Coat. Technol. 648, 174-175 (2003).

${ }^{13}$ A. C. Ferrari, "Diamond-like carbon for magnetic storage disks," Surf. Coat. Technol. 180-181, 190-206 (2004).

${ }^{14}$ M. M. Lucchese, F. Stavale, E. H. M. Ferreira, C. Vilani, M. V. O. Moutinho, R. B. Capaz, C. A. Achete, and A. Jorio, "Quantifying ion-induced defects and Raman relaxation length in graphene," Carbon 48, 1592 (2010).

${ }^{15}$ A. C. Ferrari, J. C. Meyer, V. Scardaci, C. Casiraghi, M. Lazzeri, F. Mauri, S. Piscanec, D. Jiang, K. S. Novoselov, S. Roth, and A. K. Geim, "Raman spectrum of graphene and graphene layers," Phys. Rev. Lett. 97, 187401 (2006).

${ }^{16}$ Y. Y. Wang, Z. H. Ni, T. Yu, Z. X. Shen, H. M. Wang, Y. H. Wu, W. Chen, and A. T. S. Wee, "Raman studies of monolayer graphene: The substrate effect," J. Phys. Chem. C 112, 10637-10640 (2008).

${ }^{17}$ A. Boosalis, R. Elmquist, M. Real, N. Nguyen, M. Schubert, and T. Hofmann, A Model Dielectric Function for Graphene from the Infrared to the Ultraviolet (Mater. Res. Soc. Symp. Proc. 2013), Vol. 1505.

${ }^{18}$ M. Castro, R. Cuerno, M. Nicoli, L. Vazquez, and J. G. Buijnsters, "Universality of cauliflower-like fronts: From nanoscale thin films to macroscopic plants," New J. Phys. 14, 103039 (2012).

${ }^{19}$ K. I. Bolotin, K. J. Sikes, Z. Jiang, M. Klim, G. Fudenberg, J. Hone, P. Kim, and H. L. Stormer, "Ultrahigh electron mobility in suspended graphene," Solid State Commun. 146, 351-355 (2008).

${ }^{20}$ J. Kim, M. Ishihara, Y. Koga, K. Tsugawa, and M. Hasegawa, "Low-temperature synthesis of large-area graphene-based transparent conductive films using surface wave plasma chemical vapor deposition," Appl. Phys. Lett. 98, 091502 (2011).

${ }^{21} \mathrm{~K}$. Soo et al., "Large-scale pattern growth of graphene films for stretchable transparent electrodes," Nature 457, 7230 (2009). 